7. Thele R, Gumpert H, Christensen LB, et al. Draft genome sequence of a Kluyvera intermedia isolate from a patient with a pancreatic abscess. J Glob Antimicrob Resist 2017;10:1-2.

8. Clinical and Laboratory Standards Institute. M100 - Performance Standards for Antimicrobial Susceptibility Testing. Wayne, PA: CLSI; 2018.

9. Naas T, Cuzon G, Truong H, Nordmann P. Role of IS Kpn7 and deletions in bla KPC gene expression. Antimicrob Agents Chemother 2012;56:4753-4759.

10. Fehlberg LCC, Carvalho AMC, Campana EH, Gontijo-Filho PP, Gales AC. Emergence of Klebsiella pneumoniae-producing KPC-2 carbapenemase in Paraíba, Northeastern Brazil. Brazilian J Infect Dis 2012;16:577-580.
11. Seki LM, Pereira PS, Maria da Penh AH, et al. Molecular epidemiology of KPC-2-producing Klebsiella pneumoniae isolates in Brazil: the predominance of sequence type 437. Diagn Microbiol Infect Dis 2011;70: 274-247.

12. Geffen Y, Adler A, Paikin S, et al. Detection of the plasmid-mediated KPC-2 carbapenem-hydrolysing enzyme in three unusual species of the Enterobacteriaceae family in Israel. J Antimicrob Chemother 2013;68: 719-720.

13. Wang L, Jing Y, Lai K, An J, Yang J. A Case of Biliary Tract Infection Caused by KPC-2-producing Kluyvera ascorbata. Case Rep Infect Dis 2018;2018:1-2.

\title{
Mycobacterium saskatchewanense strain associated with a chronic kidney disease patient in an Italian transplantation hospital and almost misdiagnosed as Mycobacterium tuberculosis
}

\author{
Giuseppina Di Mento BSc ${ }^{1, a}$, Anna Paola Carreca BSc ${ }^{2, a}$, Francesco Monaco BSc ${ }^{1}$, Nicola Cuscino BSc ${ }^{1}$, \\ Francesca Cardinale BSc ${ }^{1}$, Pier Giulio Conaldi MD, $\mathrm{PhD}^{1}$ and Bruno Douradinha $\mathrm{PhD}^{1,2}$ (D) \\ ${ }^{1}$ Unità di Medicina Rigenerativa e Biotecnologie Avanzate, Istituto Mediterraneo per i Trapianti e Terapie ad Alta Specializzazione, Palermo, Italy and ${ }^{2}$ Unità di \\ Medicina Rigenerativa ed Immunologia, Fondazione Ri.MED, Palermo, Italy
}

To the Editor-During an evaluation for a solid organ transplant (SOT) procedure, we identified a rare, opportunistic nontuberculous (NTM) strain, Mycobacterium saskatchewanense. The patient, who suffered from chronic renal disease, was tested for several pathogens, Mycobacterium tuberculosis included. Transplantation procedures rely on immunosuppressive therapies, which increase the success of the surgery and the survival of the patient. ${ }^{1}$ However, they also render the patients more susceptible to microbial infections, such as multidrug-resistant bacterial species like Klebsiella pneumoniae., Thus, patients require rigorous evaluations to confirm that they are not infected with pathogenic microorganisms that might compromise their health or lead to the rejection of the transplanted organ. One of the most opportunistic pathogens that can cause complications in SOT is M. tuberculosis, which infects up to $6.4 \%$ of transplant recipients in developed countries and up to $15 \%$ in areas where tuberculosis is highly endemic. ${ }^{4}$ Thus, exhaustive investigations, including the use of classical diagnostic microbiological and molecular assays are essential to ensuring that patients are fit for a SOT procedure or that they receive the correct antimicrobial therapy.

At the Istituto Mediterraneo per i Trapianti e Terapie ad Alta Specializzazione (IRCCS-ISMETT), in Palermo, Italy, a 46-yearold man with hereditary chronic renal disease requiring dialysis for the previous 2 years was evaluated for renal transplantation. He had a positive interferon- $\gamma$ release assay, suggesting prior exposure to M. tuberculosis. This patient had no previous history of tuberculosis infection, and his chest imaging did not show any signal of $M$. tuberculosis infection in the lungs. To confirm the presence of mycobacterial antigens in the blood, an ELISpot and T-SPOT.TB (Oxford Immunotec, Oxford, UK) were performed according to the manufacturer's instructions. Samples of sputum

\footnotetext{
Author for correspondence: B. Douradinha, Email: bdouradinha@fondazionerimed.com

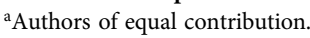

Cite this article: Di Mento G, et al. (2019). Mycobacterium saskatchewanense strain associated with a chronic kidney disease patient in an Italian transplantation hospital and almost misdiagnosed as Mycobacterium tuberculosis. Infection Control \& Hospital Epidemiology, 40: 496-497, https://doi.org/10.1017/ice.2019.6
}

and urine were also inoculated into liquid BBL mycobacteria growth indicator tubes (Becton Dickinson, Milan, Italy) according to the supplier's instructions to identify potential Mycobacteria. We obtained positive results for the presence of $M$. tuberculosis antigens in the blood and observed mycobacterial growth in the urine sample. Sputum samples were negative.

Because no M. tuberculosis was present in the patient's sputum and because this particular ELISpot has previously shown some limitations, ${ }^{5}$ we decided to extract the genomic material of this strain for sequencing. Total mycobacterial DNA was extracted using a QIAamp UCP Pathogen Mini Kit (Qiagen, Venlo, Netherlands), as specified by the kit's manual. Next-generation sequencing (NGS, full genome) and Sanger sequencing (16S rRNA gene, internal transcribed spacer (ITS1) 16S-23S and hsp65) were performed as published elsewhere. ${ }^{2,6}$ The sequences derived from both techniques showed a homology $>99 \%$ with the publicly available sequences of previously identified NTM M. saskatchewanense, from the complex $M$. simiae, ${ }^{7}$ previously only reported in the United States ${ }^{8}$ and Canada. ${ }^{6}$ This particular NTM had been isolated from a bronchiectasis patient, both from sputum and from thoracenthesis fluid, and was thought to have contributed to the deterioration of the patient. ${ }^{6}$ Genomic sequences of the M. saskatchewanense ISMETT strain were deposited in the NCBI public database (accession no. SRP149411).

The ELISpot was repeated 3 weeks later, yielding a negative result. Thus, and since we had confirmed that he was not infected with $M$. tuberculosis, the patient was not subjected to any antimycobacterial therapy. Because these interferon- $\gamma$ release assays do not allow proper distinction between colonization and infection, ${ }^{9}$ we can only assume, due to its brevity, that the patient underwent a self-resolving episode of $M$. saskatchewanense colonization.

To the best of our knowledge, this is the first time this particular NTM has been detected outside North America. We assessed its antimicrobial sensitivity to several antibiotics. The minimal inhibitory concentrations (MIC) were determined using SlowMyco Sensititre plates (Thermo Fisher Scientific, Waltham, MA), according to the 
Table 1. Minimal inhibitory concentrations (MIC) of M. saskatchewanense ISMETT strain

\begin{tabular}{lc}
\hline Antibiotic & $\mathrm{MIC}(\mu \mathrm{g} / \mathrm{mL})$ \\
\hline Amikacin & 8 \\
\hline Ciprofloxacin & 16 \\
\hline Clarithromycin & 0.5 \\
\hline Doxycycline & $>16$ \\
\hline Ethambutol & 16 \\
\hline Ethionamide & $>20$ \\
\hline Isoniazid & $>8$ \\
\hline Linezolid & 8 \\
\hline Moxifloxacin & 2 \\
\hline Rifabutin & 8 \\
\hline Rifampin & 8 \\
\hline Streptomycin & 8 \\
\hline Trimethoprim/ Sulfamethoxazole & $8 / 152$ \\
\hline
\end{tabular}

manufacturer's instructions (Table 1). Our results show an antibiotic resistance profile similar to those previously observed in Canada with their local M. saskatchewanense strains. ${ }^{6}$ Some differences were also noted; for example, the MIC for rifampicin for all Canadian strains is $0.06 \mu \mathrm{g} / \mathrm{mL}$, whereas our strain displayed a value of $8 \mu \mathrm{g} / \mathrm{mL}$ for the same antibiotic (Table 1). Although we cannot exclude the possibility that the differences observed may be due to the use of different assays, they may also indicate that the Italian M. saskatchewanense has either undergone mutations since it left North America or that its origin lies elsewhere. However, no epidemiological data about this NTM are currently available, and we can only speculate about whether it derives from the North American strains or from some other geographical location.

To the best of our knowledge, we report for the first time the detection of a $M$. saskatchewanense clinical isolate in a European health facility. Our results highlight the fact that screening assays for TB detection in blood can produce misleading results and could lead to incorrect antimicrobial therapy. Careful evaluation for mycobacterial infection must be performed, and the organism must be identified and coupled with highly discriminating techniques such as NGS, as necessary.

Author ORCIDs. Bruno Douradinha, (D) 0000-0002-9980-4505

Acknowledgments. We thank the Molecular Diagnostics at ISMETT team members for technical support and helpful insight. Informed consent of all inhouse patients is required by internal regulations before any experiments can be performed with their clinical samples.

Financial support. This study was supported by internal funding.

Conflicts of interest. All authors report no conflicts of interest relevant to this article.

\section{References}

1. Allison TL. Immunosuppressive therapy in transplantation. Nurs Clin N Am 2016;51:107-120.

2. Di Mento G, Cuscino N, Carcione C, Cardinale F, Conaldi PG, Douradinha B. Emergence of a Klebsiella pneumoniae ST392 clone harbouring KPC-3 in an Italian transplantation hospital. J Hosp Infect 2018;98:313-314.

3. Monaco F, Di Mento G, Cuscino N, Conaldi PG, Douradinha B. Infant colonisation with Escherichia coli and Klebsiella pneumoniae strains coharbouring blaOXA-48 and blaNDM-1 carbapenemases genes: a case report. Int J Antimicrob Agent 2018;52:121-122.

4. Aguado JM, Silva JT, Samanta P, Singh N. Tuberculosis and transplantation. Microbiol Spectr 2016;4:607-622.

5. Zhu C, Liu Z, Li Z, Mei S, Hu Z. The performance and limitation of T-SPOT. TB for the diagnosis of TB in a high prevalence setting. $J$ Thorac Dis 2014;6:713-719.

6. Turenne CY, Thibert L, Williams K, et al. A novel slowly growing scotochromogenic species from human clinical isolates related to Mycobacterium interjectum and Accuprobe-positive for Mycobacterium avium complex. Int J Sys Evol Microbiol 2004;54:659-667.

7. Fedrizzi T, Meehan CJ, Grottola A, et al. Genomic characterization of nontuberculous Mycobacteria. Sci Rep 2017;7:1-14.

8. Springer B, Stockman L, Teschner K, Roberts GD, Bo EC. Two-laboratory collaborative study on identification of Mycobacteria: molecular versus phenotypic methods. J Clin Microbiol 1996;34:296-303.

9. Hermansen TS, Thomsen VØ, Lillebaek T, Ravn P. Nontuberculous Mycobacteria and the performance of interferon gamma release assays in Denmark. PLoS One 2014;9:e93986-e93986.

\title{
Oseltamivir for prophylaxis of influenza in vaccinated hemodialysis patients
}

\author{
Jens T. Van Praet MD, $\mathrm{PhD}^{1}$, Sanne Steyaert $\mathrm{MD}^{1}$, Mirjam Demesmaecker ${ }^{1}$, Veerle Grootaert PharmD ${ }^{2}$ and \\ Marijke Reynders $\mathrm{MD}^{3}$ \\ ${ }^{1}$ Department of Nephrology and Infectious diseases, AZ Sint-Jan Brugge-Oostende AV, Brugge, Belgium, ${ }^{2}$ Department of Pharmacy, AZ Sint-Jan Brugge-Oostende \\ AV, Brugge, Belgium and ${ }^{3}$ Division of Medical Microbiology, Department of Laboratory Medicine, AZ Sint-Jan Brugge-Oostende AV, Brugge, Belgium
}

To the Editor-Influenza is a seasonal respiratory orthomyxoviral infection that causes major morbidity and mortality in elderly and

Author for correspondence: Jens Van Praet, Email: Jens.VanPraet@azsintjan.be

Cite this article: Van Praet JT, et al. (2019). Oseltamivir for prophylaxis of influenza in vaccinated hemodialysis patients. Infection Control \& Hospital Epidemiology, 40: 497-498, https://doi.org/10.1017/ice.2019.11 patients with chronic conditions such as end-stage renal disease. The virus is transmitted via small-particle aerosols and large droplets. ${ }^{1}$ These transmission modes are highly relevant in the context of a hemodialysis unit because patients stay in the same room for several hours and healthcare workers can facilitate the spread through inadequate hand hygiene. Serological studies indicate an 